\title{
Ionic reactivity at contacts and aging of methylammonium lead triiodide perovskite solar cell
}

\author{
Jordi Carrillo $^{1}$, Antonio Guerrero ${ }^{1}$, Sara Rahimnejad ${ }^{1,2}$, Osbel Almora ${ }^{1}$, Isaac Zarazua ${ }^{1}$, \\ Elena Mas-Marza ${ }^{1}$, Juan Bisquert ${ }^{1,3}$, and Germà Garcia-Belmonte ${ }^{1, *}$ \\ 1 Institute of Advanced Materials (INAM), Universitat Jaume I, 12071 Castelló, \\ Spain \\ 2 Department of Chemistry, Faculty of Science Yadegar-e-Imam Khomeini (RAH) \\ Shahre Rey Branch, Islamic Azad University, P.O. Box: 18155 144, Tehran, Iran \\ 3 Department of Chemistry, Faculty of Science, King Abdulaziz University, Jeddah \\ 21589, Saudi Arabia
}

Hybrid lead halide perovskites have reached very large solar to electricity power conversion efficiencies, even in some cases exceeding 20\%. The most extensively used perovskite-based solar cell configuration comprises $\mathrm{CH}_{3} \mathrm{NH}_{3} \mathrm{PbI}_{3}\left(\mathrm{MAPbI}_{3}\right)$ in combination with electron $\left(\mathrm{TiO}_{2}\right)$ and hole 2,2',7,7'-tetrakis(N,N-di-pmethoxyphenylamine)-9,9-spirobifluorene (spiro-OMeTAD) selective contacts. The recognition that the solar cell performance is heavily affected by time scale of the measurement and preconditioning procedures has raised many concerns about the stability of the device and reliability for long time operation. Mechanisms at contacts originate observable current-voltage distortions. Two types of reactivity sources have been identified here: $(i)$ weak $\mathrm{Ti}-\mathrm{I}-\mathrm{Pb}$ bonds that facilitate interfacial accommodation of moving iodine ions. This interaction produces a highly reversible capacitive current originated at the $\mathrm{TiO}_{2} / \mathrm{MAPbI}_{3}$ interface, and it does not alter steady-state photovoltaic features. (ii) An irreversible redox peak only observable after positive poling at slow scan rates. It corresponds to the chemical reaction between spiro-OMeTAD ${ }^{+}$and migrating $\mathrm{I}^{-}$which progressively reduces the hole transporting material conductivity and deteriorates solar cell performance.

*Corresponding author: G. Garcia-Belmonte, (garciag@uji.es) tel.: +34 964387538 
24 February 2016

Published: Advanced Energy Materials 2016, DOI: 10.1002/aenm.201502246 


\section{Introduction}

Hybrid lead halide perovskites have reached very large solar to electricity power conversion efficiencies, ${ }^{[1-3]}$ even in some cases exceeding $20 \% .{ }^{[4]}$ In the last two years a variety of configurations have been checked by modified cell structure, selective contacts and perovskite materials utilized. ${ }^{[5-9]}$ The most extensively used perovskitebased solar cell configuration comprises $\mathrm{CH}_{3} \mathrm{NH}_{3} \mathrm{PbI}_{3}\left(\mathrm{MAPbI}_{3}\right)$ or its analogous using chlorine precursor, $\mathrm{CH}_{3} \mathrm{NH}_{3} \mathrm{PbI}_{3-\mathrm{x}} \mathrm{Cl}_{\mathrm{x}}$, as absorber materials, in combination with electron $\left(\mathrm{TiO}_{2}\right)$ and hole 2,2',7,7'-tetrakis(N,N-di-p-methoxyphenylamine)-9,9spirobifluorene (spiro-OMeTAD) selective contacts. In spite of the spectacular advances in cell efficiency many important aspects of the experimental observations on this system are not understood. Particularly intriguing is the hysteresis features in the response of the current density-voltage $(J-V)$ curves $^{[10-13]}$ that have been related to a number of different explanations, as ferroelectric properties of the perovskite materials, ${ }^{[14-16]}$ delayed electronic trapping processes, ${ }^{[17]}$ or slow ion migration. ${ }^{[11,18,19]}$ The recognition that the solar cell performance is heavily affected by time scale of the measurement and preconditioning procedures ${ }^{[9,17,20,21]}$ has raised many concerns about the stability of the device and reliability for long time operation.

It has been recognized that interfaces between the absorber perovskite and the contacting transport layers play a central role on the solar cell operation and performance. ${ }^{[22]}$ Details of the interfaces surely depend on the synthetic methods used and materials processing. Interfacial electronic properties as energy level alignment constitute a relevant piece of knowledge that can be addressed through photoemission spectroscopies. ${ }^{[23,24]}$ From these works several recommendations for proper interface engineering are extracted. It is recognized that spiro-OMeTAD exhibits a non-optimum energy level alignment at interfaces with perovskite absorbers, while the $\mathrm{TiO}_{2}$ conduction band is in relatively fair energy alignment. ${ }^{[23]}$ The use of other contact materials of diverse electronic character (either $n$ - or $p$-type, and insulating) have revealed that the Fermi level within $\mathrm{MAPbI}_{3}$ is strongly influenced by the substrate conductivity type. ${ }^{[24]}$ In connection with the electronic interfacial properties, recent electrical measurements have shown that the potential drop concentrates near the $\mathrm{TiO}_{2} / \mathrm{MAPbI}_{3}$ contact forming a sort of $p$ - $n$ junction. ${ }^{[25,26]}$ But interfaces evolve during solar cell operation, presumably by the presence of moving ions, in such a way that the connection between photoemission spectroscopy findings and the reported $J-V$ distortions needs of complementary analysis. Moreover in-situ measurements 
with solar cells under operation require of specific methods different from photoemission spectroscopies. Evolving reactivity and chemical degradation at interfaces can in turn be addressed by $J-V$ analysis of selected structures in the light of electrochemically-inspired methods. ${ }^{[27,28]}$

Here we provide insights about time-dependent photovoltaic operation by discerning the locus of hysteresis response. We show that contact phenomena distort the $J-V$ curve. Using different cell structures, we differentiate for the first time the different types of interactions of the standard electrode contacts. They display qualitatively different sources of reactivity at the interface between $\mathrm{MAPbI}_{3}$ and the transporting layers. At $\mathrm{TiO}_{2}$ contact we obtain reversible capacitive currents. On the other hand irreversible ionic reaction occurs between mobile ions in $\mathrm{MAPbI}_{3}$ and spiro-OMeTAD organic hole extracting layer. Only the latter irreversible behavior may cause significant long term aging by reduction of spiro-OMeTAD conductivity, and it is therefore a key point for engineering of the solar cell towards long time robust operation.

Recent experimental work shows a connection between capacitive current and hysteresis behavior in hybrid lead halide perovskites. ${ }^{[27]}$ The microscopic phenomena responsible for capacitive dark currents is the ionic electrode polarization, similar to double layer capacitive effects, while the perovskite absorber layer behaves as a solid electrolyte able to interact with the contacting transport layers. However, it has not been determined which contact interface, either $\mathrm{MAPbI}_{3} / \mathrm{TiO}_{2}, \mathrm{MAPbI}_{3} /$ spiro-OMeTAD, or both, originates the observed capacitive response even in dark conditions. In this study we report scans of current-voltage curves, both in solar cells of planar structure $\mathrm{FTO} / \mathrm{TiO}_{2} / \mathrm{MAPbI}_{3} /$ spiro-OMeTAD/Au (Figure 1a), and in planar perovskite layers with symmetric contacts of both types, electron and hole extracting electrodes. The purpose of the investigation is to differentiate which contact or part of the solar cell is causing specific hysteresis of irreversible features in current-voltage curves causing detrimental effects on the solar cell long time performance.

\section{Results and Discussion}

Figure $1 \mathrm{~b}$ shows that capacitive currents dominate current-voltage response in the dark within the central voltage window. In addition one observes injection currents at positive voltages $(>0.5 \mathrm{~V})$ and leakage currents at negative bias $(<-0.5 \mathrm{~V})$. Here the diode convention (common in Electronics) is used: positive bias implies negative voltage at the cathode $\left(\mathrm{ITO} / \mathrm{TiO}_{2}\right)$ contact, in such a way that electrons are injected 
through the cathode and holes through the anode (Au/ spiro-OMeTAD). It is remarkable how the hysteresis disappears when approaching steady-state conditions, being highly reversible as the signal level is independent on the sweep direction.

Positive poling ( $1 \mathrm{~V}$ during $5 \mathrm{~min}$ ) alters the quasi steady-state response (low scan rate) as observed in Figure 1c. However, cycled negative poling does not produce any effect on previous signals obtained for positive poling experiments (see Figure S1). Figure 1c shows that a positive peak initially developed at $\sim 0.2-0.3 \mathrm{~V}$ is enlarged for successive poling cycles. This additional signal is only partially compensated by a negative peak appearing at $\sim-0.1-0.0 \mathrm{~V}$. In addition injection current at forward bias is reduced upon cycling (inset of Figure 1c). We conclude that polarization and slow scan rates produce a partially reversible peak with an incremental effect indicating stable chemical interactions and forward current reduction at large bias. We note that this last behaviour is different from the effect produced by bias pre-conditioning under illumination. $^{[29]}$ It causes a non-capacitive distortion of the current-voltage characteristics by ion-induced contact barrier modification. 


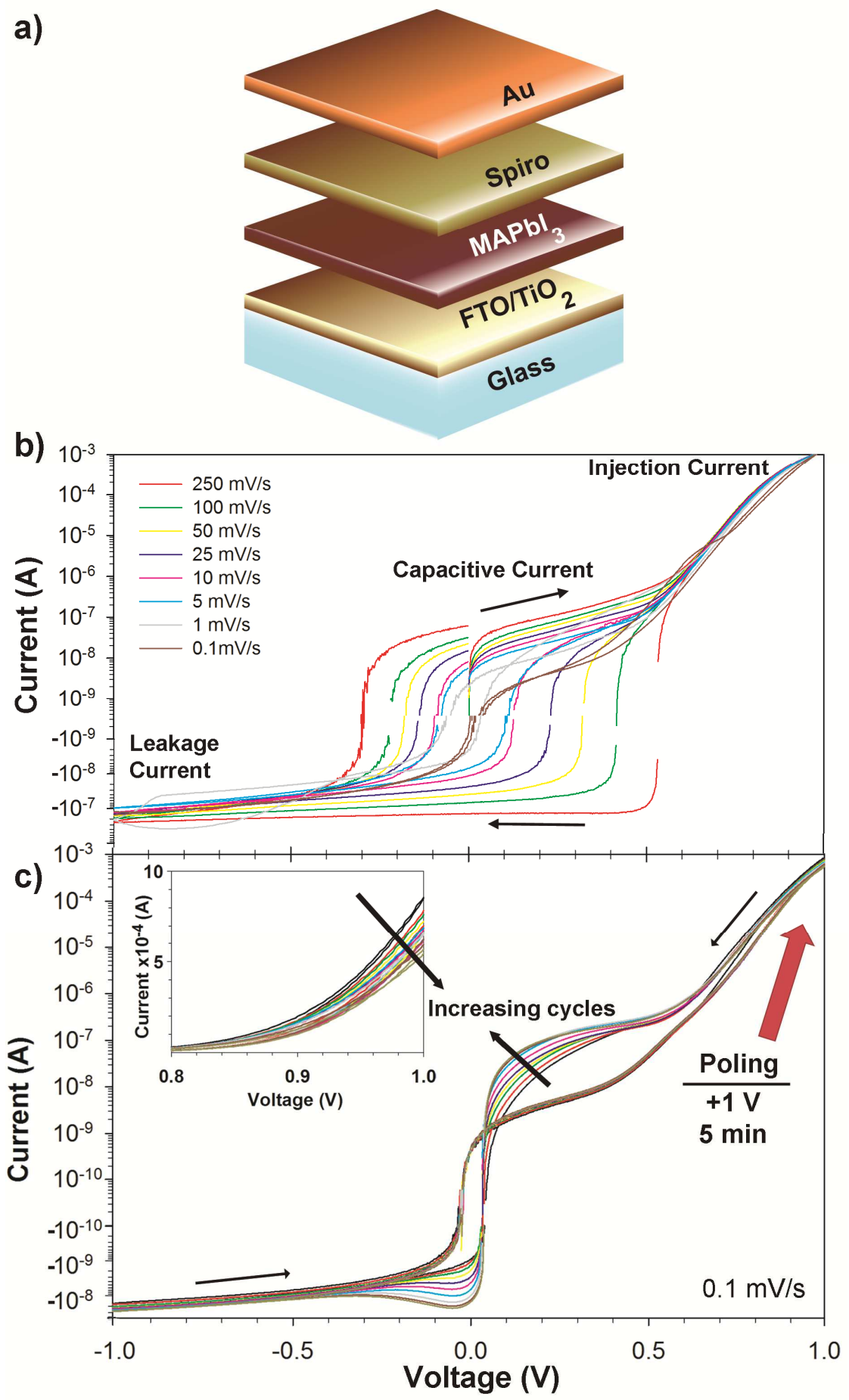

Figure 1. a) Structure of the planar solar cells checked. Thin $\mathrm{MAPbI}_{3}$ layer $(200 \mathrm{~nm})$ is sandwiched between $\mathrm{TiO}_{2}$ and spiro-OMeTAD selective contacts. b) Dark currents measured at different scan rates both for positive and negative sweep. Three different mechanisms are identified: at $V>0.5 \mathrm{~V}$ operation currents caused by carrier injection, $V<-0.5 \mathrm{~V}$ leakage currents, and central voltage interval shows the presence of scanrate dependent, capacitive currents. The slowest scan rate approaches steady-state response. c) Cycled experiments including positive poling during $5 \mathrm{~min}$. Positive scan (no poling) reproduce steady-state behavior. Negative scans induce incremental redox peaks after successive cycles. The process is only partially reversible. In the inset: injection current at forward bias decreases with cycling. 
Samples of symmetrical devices are useful to investigate separate electrode effects occurring in solar cells. As explained in the Experimental section, $500 \mu \mathrm{m}$-thick $\mathrm{MAPbI}_{3}$ pellets are prepared and sandwiched between contact electrodes comprising transport layers (see Figure S2 and S3 for XDR and SEM images). Symmetrical samples of structure $\mathrm{FTO} / \mathrm{TiO}_{2} / \mathrm{MAPbI}_{3} / \mathrm{TiO}_{2} / \mathrm{FTO}$ (Figure 2a) and $\mathrm{Au} /$ spiro$\mathrm{OMeTAD} / \mathrm{MAPbI}_{3} /$ spiro-OMeTAD/Au (Figure 3a) are obtained. Figure $2 \mathrm{c}$ shows the response when two $\mathrm{TiO}_{2}$ contact layers are used. Perfect square-like signals clearly indicate the occurrence of capacitive currents comparable to those obtained for complete solar cells $\left(0.1 \mu \mathrm{A}\right.$ for $250 \mathrm{mV} \mathrm{s}^{-1}$ scan rate) in Figure $1 \mathrm{~b}$. The fact that both solar cells and symmetrical devices yield similar current level, despite the huge difference in $\mathrm{MAPbI}_{3}$ layer thickness (200 $\mathrm{nm}$ for thin films and $500 \mu \mathrm{m}$ for pellets), corroborates the interfacial origin of the response. Figure $2 \mathrm{~d}$ shows a linear dependence between current level and scan rate which allows extracting capacitive values approximately equal to $\sim 2 \mu \mathrm{F} \mathrm{cm}^{-2}$, again in good agreement with typical double layer capacitors $\left(\sim 10 \mu \mathrm{F} \mathrm{cm}^{-2}\right)$. It is also observed that polarization does not induce any additional feature in the form of redox peaks (Figure S4). It is concluded that dark capacitive currents are originated by electrode polarization at $\mathrm{TiO}_{2} / \mathrm{MAPbI}_{3}$ interfaces. 

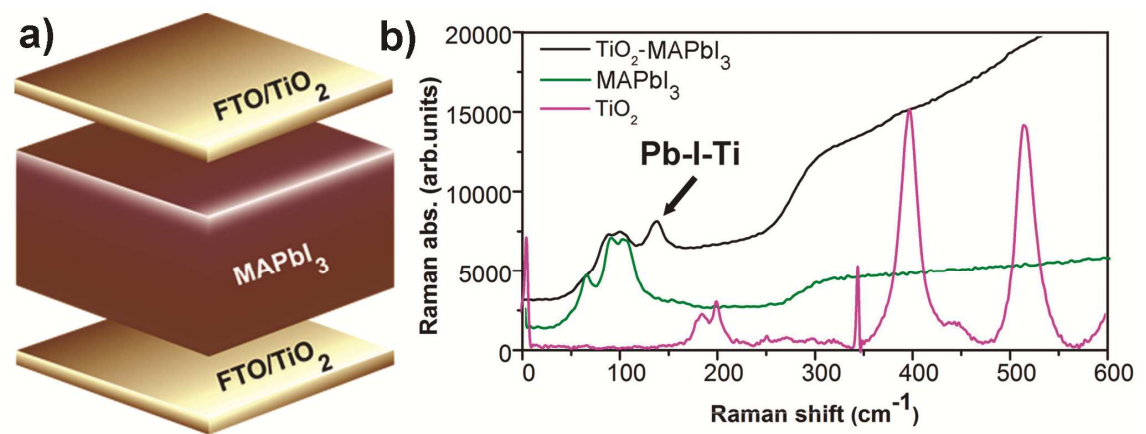

c)

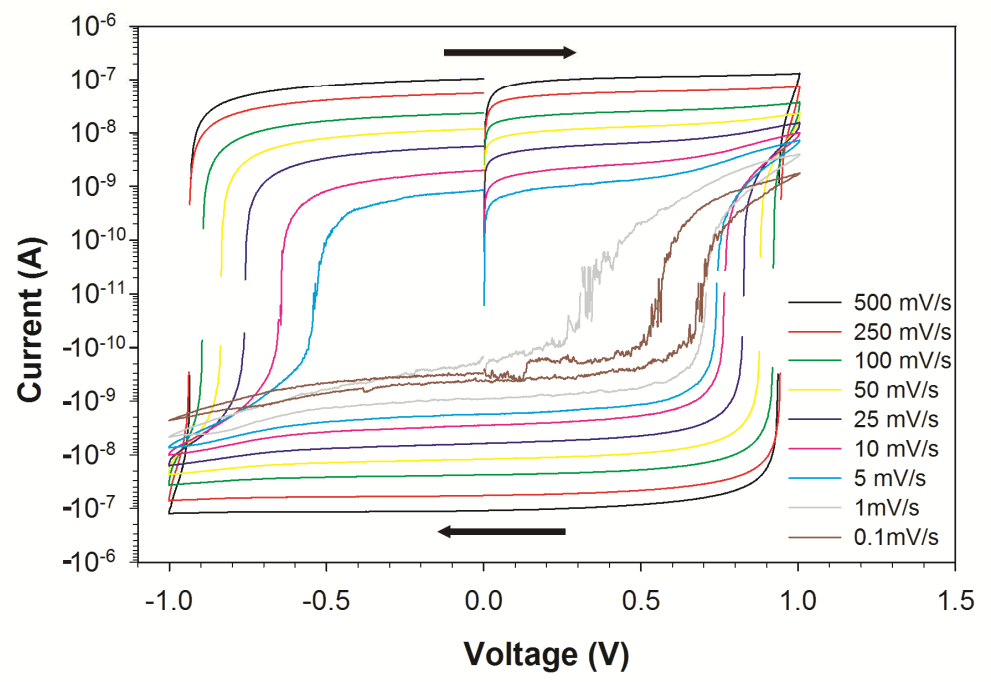

d)

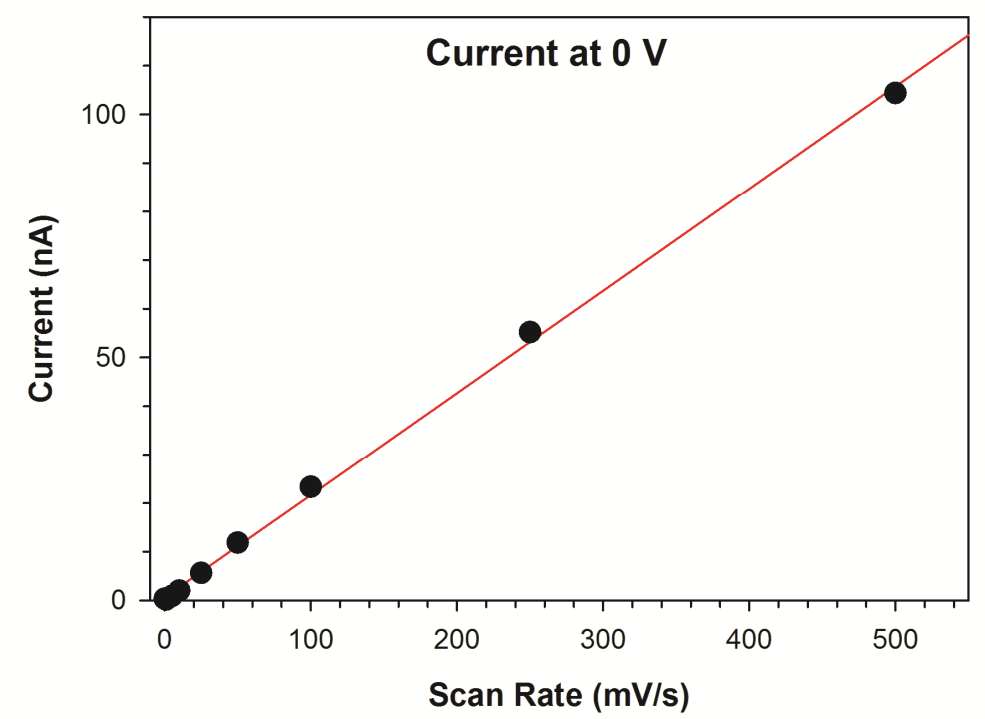

Figure 2. a) Structure of $\mathrm{MAPbI}_{3}(500 \mu \mathrm{m})$ symmetric devices with $\mathrm{TiO}_{2}$ contact layers. b) Raman spectra illustrating the formation of $\mathrm{Ti}-\mathrm{I}-\mathrm{Pb}$ bonds upon reaction between MAI and $\mathrm{PbI}_{2}$. c) Capacitive currents exhibited by $\mathrm{TiO}_{2} / \mathrm{MAPbI}_{3} / \mathrm{TiO}_{2}$ samples. Perfect square-like, scan rate-dependent responses are obtained indicating electrode capacitance behavior. d) Current level at $0 \mathrm{~V}$ as a function of the scan rate. Linear slope corresponds to $\sim 2 \mu \mathrm{F} \mathrm{cm}^{-2}$.

The $J-V$ response changes completely when Au/spiro-OMeTAD/MAPbI $3 /$ spiro$\mathrm{OMeTad} / \mathrm{Au}$ samples (Figure 3a) are analyzed. Figure $3 \mathrm{c}$ shows that only ohmic currents are observed. Because of the thick perovskite layer used it is expected that 
currents (presumably electronic leakage currents) are mainly governed by the bulk transport properties. In any case the response is observed to be scan-rate independent. In order to verify if additional signals are present, in similarity to redox peaks of Figure 1c, it is necessary to explore in detail low scan rate signals. Inset of Figure $3 c$ shows in linear scale a current peak that slightly distorts the base ohmic behavior, only observable at $0.1 \mathrm{mV} \mathrm{s}^{-1}$. Therefore analysis of symmetrical devices using holetransporting layers suggests that $\mathrm{MAPbI}_{3}$ interacts with spiro-OMeTAD producing detectable signals. spiro-OMeTaAD is prepared without lithium salts to exclude $\mathrm{Li}^{+}$ migration and de-doping. Results of spiro-OMeTaAD layers processed with lithium bis(trifluoromethylsulphonyl)imide are shown in Figure S5 (SI).

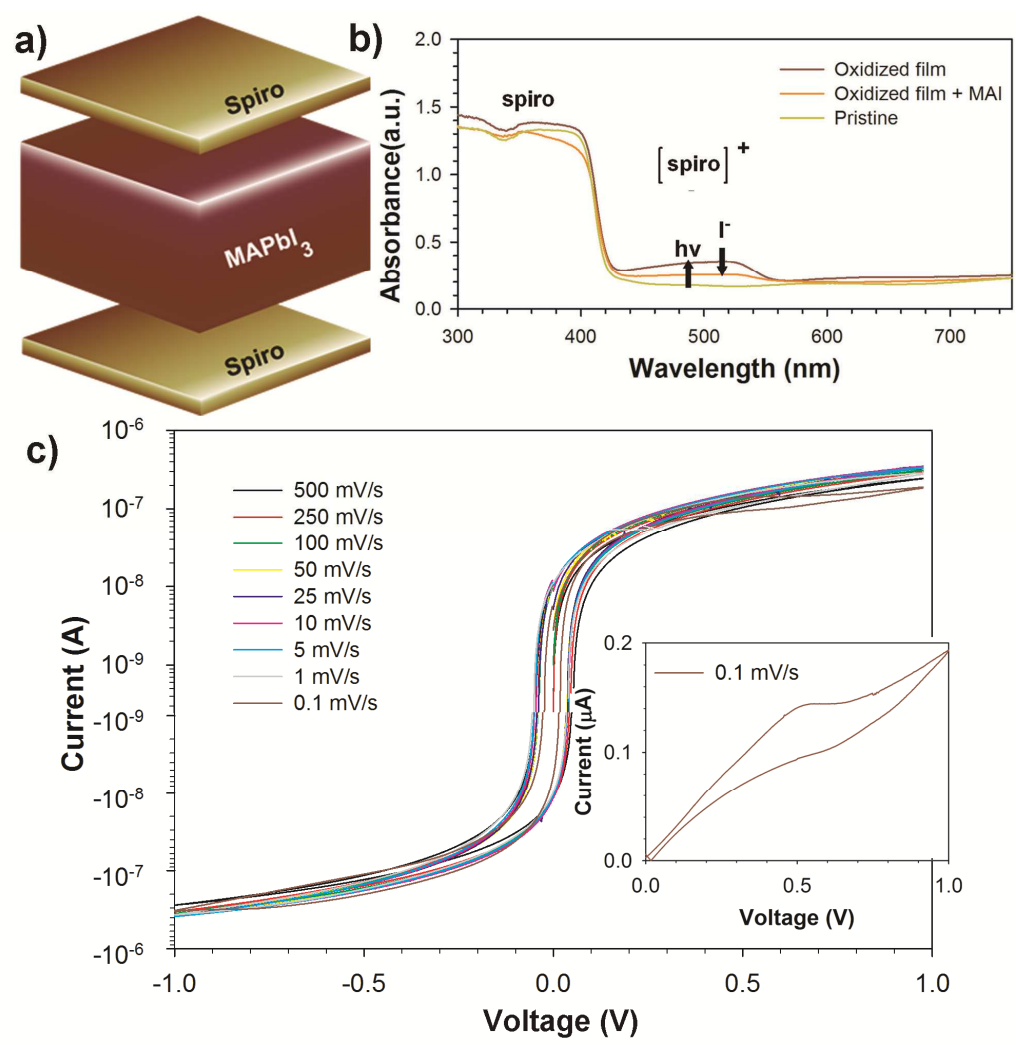

Figure 3. a) Structure of $\mathrm{MAPbI}_{3}(500 \mu \mathrm{m})$ symmetric devices with spiro-OMeTAD contact layers. b) Absorption spectra of films of pristine and oxidized spiro-OMeTAD layers, and the reduction of the polaronic band $(\sim 500 \mathrm{~nm})$ by reaction with a source of $\mathrm{I}^{-}$ . c) Current-voltage characteristics of spiro-OMeTAD/MAPbI $\mathrm{I}_{3} /$ spiro-OMeTaAD samples exhibiting ohmic, scan rate-independent behavior. At slowest scan rates (inset) $\mathrm{MAPbI}_{3}$ interacts with spiro-OMeTAD producing detectable signals. spiro-OMeTaAD is prepared without lithium salts to exclude $\mathrm{Li}^{+}$migration and de-doping. 
Our experiments have led us to separate two types of contact layer/perovskite interactions: either highly reversible one, producing the capacitive response originated at the $\mathrm{TiO}_{2} / \mathrm{MAPbI}_{3}$ interface, or in the form of a well-differentiate redox peak related to the spiro-OMeTAD/MAPbI 3 contact. Reversibility indicates a weak chemical interaction as those occurring in polarized surfaces forming an interface charge of adsorbed ions. A computational study of the $\mathrm{TiO}_{2} / \mathrm{MAPbI}_{3}$ interface by De Angelis and coworkers, ${ }^{[30]}$ indicates that $\mathrm{TiO}_{2} /$ perovskite interaction occurs mainly through the binding of perovskite iodine atoms to under-coordinated $\mathrm{Ti}(\mathrm{IV})$ atoms of the $\mathrm{TiO}_{2}$ surface. Moreover the two materials interact only weakly, as seen by the little hybridization found between the perovskite $\mathrm{Pb} s-p$ states and the $\mathrm{Ti} d$ orbitals constituting the $\mathrm{TiO}_{2}$ conduction band. As a result an electrical dipole is formed with $\sim 4.8 \mathrm{D}$ per $\mathrm{nm}^{2}$ of $\mathrm{TiO}_{2}$ surface. The identification of the $\mathrm{TiO}_{2} / \mathrm{MAPbI}_{3}$ chemical bonds along with evidences of easy iodine (defect) migration in perovskite-like materials ${ }^{[31,32]}$ suggests a mechanism able to explain the observation of capacitive currents of Figure $1 \mathrm{~b}$ and Figure $2 \mathrm{c}$ by the reversible and facile interfacial ionic accommodation promoted by moving $\mathrm{I}^{-}$.

The direct reaction between $\mathrm{MAPbI}_{3}$ and commercially available $\mathrm{TiO}_{2}$ nanoparticles has been investigated here to verify the formation of $\mathrm{Ti}-\mathrm{I}-\mathrm{Pb}$ bonds. The perovskite material has been synthesized by mixing MAI and $\mathrm{PbI}_{2}$ (1:1) in DMF followed by solvent evaporation (see Experimental). By using Raman spectroscopy Ti-I-Pb bonds are identified in the product (Figure 2b). Sign of hydrolysis is not observed as the characteristic band at $215 \mathrm{~cm}^{-1}$ for $\mathrm{PbI}_{2}$ is not present. ${ }^{[33]}$ When Raman spectra of the product are compared with reagents a new band at $137 \mathrm{~cm}^{-1}$ appears which suggests evidence for $\mathrm{Ti}-\mathrm{I}-\mathrm{Pb}$ bond generation without the presence of an applied bias as in the case of devices. The observed capacitive current is then understood by means of the diagram shown in Figure 4. At positive applied bias iodide migrates towards the spiroOMeTAD contact, a positive charge is generated at $\mathrm{MAPbI}_{3}$ interface in close contact to the $\mathrm{TiO}_{2}$ layer and charge is compensated by the injection and accumulations of electrons at $\mathrm{TiO}_{2}$. Finally, at negative bias $\mathrm{I}^{-}$is accumulated at $\mathrm{MAPbI}_{3}$ layer contacting $\mathrm{TiO}_{2}$. Ti-I-Pb bonds easily accommodate excess or defect ionic charge in a highly reversible way causing the capacitive currents. 
a) Positive bias

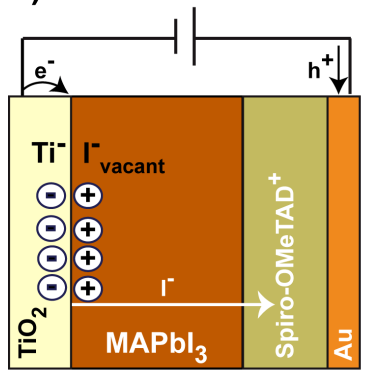

b)

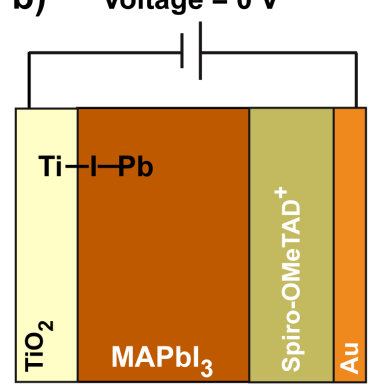

c) Negative bias

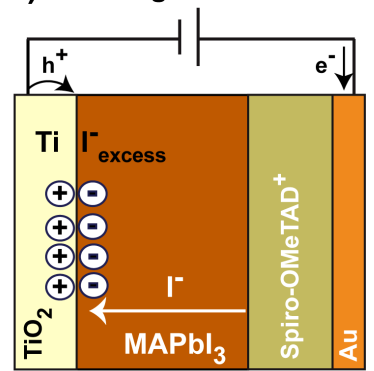

Figure 4. Diagram representing iodide migration and chemical species present at the interfaces. a) At positive bias iodine ions are forced to migrate towards the hole selective contact where the reaction with spiro-OMeTAD ${ }^{+}$occurs. Iodine defective layer is formed at the $\mathrm{TiO}_{2} / \mathrm{MAPbI}_{3}$ interface. b) At zero bias neutral case appears. c) At negative bias spiro-OMeTAD only partially returns to its oxidized, conductive state. Iodine ions accumulate at the $\mathrm{TiO}_{2}$.

A totally different scenario is observed at the spiro-OMeTAD/MAPbI ${ }_{3}$ interfaces. Spiro-OMeTad requires partial oxidation in order to work efficiently as a hole extraction layer. ${ }^{[34]}$ Indeed, photooxidation readily takes place under illumination with oxidized spiro-OMeTAD ${ }^{+}$concentrations observed above $10 \%$ during solar cell testing under ambient conditions. Partially oxidized spiro-OMeTAD molecules is known to increase hole conductivity of the layer. ${ }^{[35,36]}$ Here, iodide migration and reaction with spiro-OMeTAD layer is monitored by absorption measurements (Figure 3b). A pristine spiro-OMeTAD film only shows a characteristic band between 300-420 nm. After oxidation in the presence of oxygen the polaronic band corresponding to spiro$\mathrm{OMeTAD}^{+}$at about $500 \mathrm{~nm}$ becomes evident. This polaronic species is stabilized by the presence of weakly coordinating anions as those present in the additives (TFSI) to maintain electroneutrality. However, if the film is treated with a source of $\mathrm{I}^{-}$ions such as methylammonium iodide (MAI) the intensity of the polaronic band decreases dramatically. This result is not surprising as iodide ions are highly coordinating. In order to rule out the effect of LiTFSI, similar experiments have been carried out in the absence of this additive (see Figure S6, SI). By reaction of spiro-OMeTAD in solution with oxidizing reagent containing a poorly nucleophilic anion $\left(\mathrm{NOBF}_{4}\right)$, the polaronic band appears at $500 \mathrm{~nm}$, again confirming that the band corresponds to the spiroOMeTAD polaron. Recovery of a neutral species with a higher extinction coefficient by further reaction with MAI is again observed. Overall, the chemical reaction can be expressed as in Scheme 1.

$$
\text { spiro }-\mathrm{OMeTAD}^{+}+\mathrm{I}^{-} \rightarrow \text { spiro }-\mathrm{OMeTAD}-\mathrm{I}
$$


Although other reactions producing $\mathrm{I}_{2}$ evolution and neutral spiro-OMeTAD molecules cannot be discarded. As discussed previously, the redox peak in Figure 1c enlarges after each positive poling cycle. One can then imagine a situation as that drawn in Figure 4: at positive poling iodine ions migrate to the spiro-OMeTAD contact, and intercalate into the hole transport layer. This promotes reaction with previously oxidized spiroOMeTAD molecules. By this way spiro-OMeTAD returns to its neutral state reducing the $p$-doped character of the hole contact layer. Reaction shown in Scheme 1 occurs in a highly irreversible manner. This observation entails a progressive reduction in the conductive properties of the spiro-OMeTAD layer that finally produces a detrimental effect on the photovoltaic operation. This last mechanism agrees with the progressive reduction in the current at higher positive bias observed in the inset of Figure 1d. As spiro-OMeTAD layer conductivity is reduced injection current is limited upon cycling.

\section{Conclusion}

In summary two types of reactivity sources have been identified: (a) weak Ti-I-Pb bonds that facilitate interfacial accommodation of moving iodine ions. This interaction produces a highly reversible capacitive current originated at the $\mathrm{TiO}_{2} / \mathrm{MAPbI}_{3}$ interface. This mechanism does not alter steady-state photovoltaic features. $(b)$ an irreversible redox peak only observable after positive poling at slow scan rates. It corresponds to the chemical reaction between spiro-OMeTAD ${ }^{+}$and migrating $\mathrm{I}^{-}$which progressively reduces the hole transporting material conductivity and deteriorates solar cell performance. We remark that under operation, large photovoltage $(\sim 1 \mathrm{~V})$ is expected to occur for perovskite solar cells. Positive voltage causes ion movement and as a consequence aging effects through contact layer reaction. It is therefore essential to draw attention to chemical interactions between absorber perovskite and contact layers if this technology aims to reach long term stability.

\section{Experimental Section}

Solar cells: Substrate preparation. Fluorine doped tin oxide (FTO) coated glass substrates ( $25 \times 25 \mathrm{~mm}$, Pilkington TEC15, $\sim 15 \Omega / \mathrm{sq}$ resistance) were etched with zinc powder and $\mathrm{HCl}(2 \mathrm{M})$ to obtain $0.224 \mathrm{~cm}^{2}$ of active electrode area. The substrates were 
cleaned with soap (Hellmanex) and rinsed with milliQ water and ethanol. Then, the sheets were sonicated for 15 minutes in a solution of acetone: isopropanol $(1: 1 \mathrm{v} / \mathrm{v})$, rinsed with ethanol and dried with compressed air. After that, a UV/ozone treatment

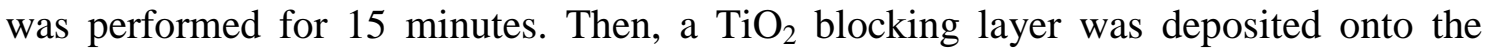
substrates by spray pyrolysis at $450^{\circ} \mathrm{C}$, using a titanium diisopropoxide bis(acetylacetonate) (75\% in isopropanol, Sigma-Aldrich) solution diluted in ethanol $(1: 39, \mathrm{v} / \mathrm{v})$, with oxygen as carrier gas. After the spraying process the films were kept at $450{ }^{\circ} \mathrm{C}$ for 5 minutes.

Mesoporous $\mathrm{TiO}_{2}$ layer. When needed, a mesoporous $\mathrm{TiO}_{2}$ layer was deposited by spin coating at $4000 \mathrm{rpm}$ during $60 \mathrm{~s}$ using a $\mathrm{TiO}_{2}$ paste (Dyesol 18NRT, $20 \mathrm{~nm}$ average particle size) diluted in terpineol (1:3, weight ratio). After drying at $80{ }^{\circ} \mathrm{C}$, the $\mathrm{TiO}_{2}$ layers were heated at $450{ }^{\circ} \mathrm{C}$ for $30 \mathrm{~min}$ and cooled to room temperature. The thickness determined by Scanning Electron Microscopy was $\sim 200 \mathrm{~nm}$.

Perovskite deposition. $100 \mu \mathrm{l}$ of the perovskite precursor solution (2.64 mmol of $\mathrm{CH}_{3} \mathrm{NH}_{3} \mathrm{I}$ and $0.88 \mathrm{mmol}$ of $\mathrm{PbCl}_{2} 1 \mathrm{~mL}$ of DMF) was spin-coated inside the glove box at 2000 r.p.m. for 60 s. After the deposition, the substrate was kept at $100^{\circ} \mathrm{C}$ for $10 \mathrm{~min}$. Next, the substrates were heated at $100^{\circ} \mathrm{C}$ during 1 hour in an oven under air stream.

Hole transport layer deposition. A 300-400 nm-thick of HTM was deposited on top of the perovskite substrates by spin coating at 4000 r.p.m for $30 \mathrm{~s}$ under air conditions, using $100 \mu \mathrm{L}$ of spiro-OMeTAD solution. The spiro-OMeTAD solution was prepared by dissolving $72.3 \mathrm{mg}$ of (2,2',7,7'-tetrakis(N,N'-di-p-methoxyphenylamine)-9,9'spirobifluorene) and $28.8 \mu \mathrm{L}$ of 4-tert-butylpyridine in $1 \mathrm{~mL}$ of chlorobenzene. When needed $17.5 \mu \mathrm{L}$ of a stock solution of $520 \mathrm{mg} / \mathrm{mL}$ of lithium bis(trifluoromethylsulphonyl)imide in acetonitrile was also added to the spiroOMeTAD solution.

Gold electrode deposition. The deposition of $60 \mathrm{~nm}$ of gold was performed by thermal evaporation under ultrahigh vacuum conditions, using a commercial MBraun vacuum chamber. Before beginning the evaporation the chamber was evacuated until pressure of $1 \cdot 10^{-6}$ mbar.

Film preparation: $F T O / T i O_{2}$ compact layer films. A compact $\mathrm{TiO}_{2}$ layer was deposited onto the cleaned substrates, by spray pyrolysis at $450^{\circ} \mathrm{C}$, using a titanium 
diisopropoxide bis(acetylacetonate) (75\% in isopropanol, Sigma-Aldrich) solution diluted in ethanol $(1: 39, \mathrm{v} / \mathrm{v})$, with oxygen as carrier gas. After the spraying process the films were kept at $450{ }^{\circ} \mathrm{C}$ for 5 minutes.

FTO/spiro-OMeTAD films. A 300-400 nm-thick of HTM was deposited on top of the cleaned substrates by spin coating at 4000 r.p.m for $30 \mathrm{~s}$ under air conditions, using 100 $\mu \mathrm{L}$ of spiro-OMeTAD solution. The spiro-OMeTAD solution was prepared by dissolving $\quad 72.3 \mathrm{mg}$ of $\left(2,2^{\prime}, 7,7^{\prime}\right.$-tetrakis(N,N'-di-p-methoxyphenylamine)-9,9'spirobifluorene) and $28.8 \mu \mathrm{L}$ of 4-tert-butylpyridine in $1 \mathrm{~mL}$ of chlorobenzene. When needed $17.5 \mu \mathrm{L}$ of a stock solution of $520 \mathrm{mg} / \mathrm{mL}$ of lithium bis(trifluoromethylsulphonyl)imide in acetonitrile was also added to the spiroOMeTAD solution.

$\mathrm{CH}_{3} \mathrm{NH}_{3} \mathrm{PbI}_{3}$ pellets: $\mathrm{CH}_{3} \mathrm{NH}_{3} \mathrm{PbI}_{3}$ was synthesised by evaporation of DMF in a solution containing stoichiometric amounts of lead iodide (1 .00 g, 1 eq) and methyl ammonium iodide $(0.345 \mathrm{~g}, 1 \mathrm{eq})$. The mixture was placed in an open crystallization dish into a well-ventilated oven at $130{ }^{\circ} \mathrm{C}$ during $3 \mathrm{~h}$, the mixture was stirred every $30 \mathrm{~min}$. The black powder was characterized by XRD to confirm the pure perovskite crystallographic form (Figure S2, SI). Alternatively, SEM analysis shows aspect of perovskite powder (Figure S3, SI). Films were prepared by pressing at 6 ton $\mathrm{CH}_{3} \mathrm{NH}_{3} \mathrm{PbI}_{3}$ powder $(0.25 \mathrm{~g})$ into a $13 \mathrm{~mm}$ pellet die. Thickness of the pellet prepared in this manner range from 500-600 $\mu \mathrm{m}$ according to SEM (Figure S3, SI).

Assembly of symmetric devices: Symmetric devices were prepared by placing a perovskite film between the outer contacts, either $\mathrm{FTO} / \mathrm{TiO}_{2}$ or $\mathrm{FTO}$ / spiro-OMeTAD, and the sandwich structure was clamped to ensure a good electrical contact. The contacts were created using crocodile clips.

Solar cells characterization: The Incident Photon to Current Efficiency (IPCE) were performed using a Xenon lamp power source coupled with a monochromator controlled by a computer. Photocurrent was measured using an optical power meter 70310 from Oriel Instruments and a Si photodiode to calibrate the system. Current density-voltage $(J-V)$ curves were performed under 1 sun illumination $\left(100 \mathrm{~mW} \mathrm{~cm}^{-2}\right)$ using a Xenon arc lamp simulator (Sun 2000, ABET Technologies) with an AM 1.5 G spectral filter and a Keithley 2400, previously calibrated with an NREL Si solar cell (Figure S7, SI). All the 
measurements were performed with an opaque mask of $0.11 \mathrm{~cm}^{2}$ and without encapsulation. The dark current-voltage and chronoamperometric curves were obtained by the use of a potentiostat/galvanostat (Autolab PGSTAT20). The initial potential was established at $0 \mathrm{~V}$ whereas the voltage ranged from $1 \mathrm{~V}$ to $-1 \mathrm{~V}$. These experiments were carried out after the IPCE measurement and chronoamperometric experiments, in order to study their electrochemical behaviour previous and after the polarization of the solar cell. The chronoamperometric curves were performed applying a voltage of $+1 \mathrm{~V}$ (or -1 V) during 5 and $15 \mathrm{~min}$. The dark conditions were obtained by coating the solar cells completely with an aluminium foil. The optical absorption spectra of the active layers were recorded by a Cary 500 Scan VARIAN spectrophotometer in the 300-2000 nm range using an integrating sphere. Oxidized spiro-OMeTAD films were generated by keeping deposited film in the dark under ambient conditions over a period of one month. The film was treated with a solution of methyl ammonium iodide $(5 \mathrm{mM})$ in isopropanol. The absorption band corresponding to oxidized spiro-OMeTAD reduces its intensity indicating that a source of nucleophilic atoms such as iodide $\left(\mathrm{I}^{-}\right)$can react with oxidized spiro-OMeTAD to recover neutral species. For Raman experiments the perovskite material has been synthesized by mixing MAI and $\mathrm{PbI}_{2}(1: 1)$ in $\mathrm{DMF}$ as solvent followed by solvent evaporation. Alternatively, the chemical reaction is carried out in ethanol, as poorly solubilizing solvent, with stirring at R.T. Relatively high energy $(80 \mathrm{~mW})$ and very low excitation times $(1 \mathrm{~s})$ are used together with a holographic filter with a cutoff edge at $70 \mathrm{~cm}^{-1}$. Raman spectroscopy measurements were carried out with a dispersive spectrometer NRS-3100 (Jasco).

\section{Acknowledgements}

We thank financial support by MINECO of Spain under project (MAT2013-47192C3-1-R), and Generalitat Valenciana (Prometeo/2014/020). A.G. would like to thank the Spanish Ministerio de Economía y Competitividad for a Ramón y Cajal Fellowship (RYC-2014-16809).

\section{References}

[1] N.-G. Park, The Journal of Physical Chemistry Letters 2013, 4, 2423.

[2] H. J. Snaith, The Journal of Physical Chemistry Letters 2013, 4, 3623.

[3] N. J. Jeon, J. H. Noh, Y. C. Kim, W. S. Yang, S. Ryu, S. I. Seok, Nat. Mater. 2014, 13, 897.

[4] M. A. Green, K. Emery, Y. Hishikawa, W. Warta, E. D. Dunlop, Prog. Photovolt: Res. Appl. 2015, 23, 1.

[5] K. Wojciechowski, S. D. Stranks, A. Abate, G. Sadoughi, A. Sadhanala, N. Kopidakis, G. Rumbles, C.-Z. Li, R. H. Friend, A. K.-Y. Jen, H. J. Snaith, ACS Nano 2014, 8, 12701. 
[6] G. A. Sepalage, S. Meyer, A. Pascoe, A. D. Scully, F. Huang, U. Bach, Y.-B. Cheng, L. Spiccia, Adv. Funct. Mater. 2015, 25, 5650.

[7] X. Xu, Z. Liu, Z. Zuo, M. Zhang, Z. Zhao, Y. Shen, H. Zhou, Q. Chen, Y. Yang, M. Wang, Nano Letters 2015, 15, 2402-2408.

[8] O. Malinkiewicz, A. Yella, Y. H. Lee, G. M. Espallargas, M. Graetzel, M. K. Nazeeruddin, H. J. Bolink, Nature Photonics 2014, 8, 128.

[9] T.-Y. Yang, G. Gregori, N. Pellet, M. Grätzel, J. Maier, Angew. Chem. 2015, 127, 8016

[10] H. J. Snaith, A. Abate, J. M. Ball, G. E. Eperon, T. Leijtens, N. Kimberly, S. D. Stranks, J. T.-W. Wang, K. Wojciechowski, W. Zhang, N. K. Noel, The Journal of Physical Chemistry Letters 2014, 5, 1511.

[11] E. L. Unger, E. T. Hoke, C. D. Bailie, W. H. Nguyen, A. R. Bowring, T. Heumüller, M. G. Christoforod, M. D. McGehee, Energy \& Environmental Science 2014, 7, 3690.

[12] N. J. Jeon, J. H. Noh, Y. C. Kim, W. S. Yang, S. Ryu, S. I. Seok, Nature Materials 2014, 13, 897-903.

[13] R. S. Sanchez, V. Gonzalez-Pedro, J.-W. Lee, N.-G. Park, Y. S. Kang, I. Mora-Sero, J. Bisquert, Journal of Physical Chemistry Letters 2014, 5, 2357-2363.

[14] J. M. Frost, K. T. Butler, A. Walsh, APL Mater. 2014, 2, 081506.

[15] J. Wei, Y. Zhao, H. Li, G. Li, J. Pan, D. Xu, Q. Zhao, D. Yu, Journal of Physical Chemistry Letters 2014, 5, 3937-3945.

[16] H.-W. Chen, N. Sakai, M. Ikegami, T. Miyasaka, Journal of Physical Chemistry Letters 2015, 6, 164-169.

[17] Y. Shao, Z. Xiao, C. Bi, Y. Yuan, J. Huang, Nat. Commun. 2014, 5, 5784.

[18] A. Dualeh, T. Moehl, N. Tétreault, J. Teuscher, P. Gao, M. K. Nazeeruddin, M. Grätzel, ACS Nano 2014, 8, 362.

[19] Z. Xiao, Y. Yuan, Y. Shao, Q. Wang, Q. Dong, C. Bi, P. Sharma, A. Gruverman, J. Huang, Nature Materials 2015, 14, 193.

[20] R. Gottesman, E. Haltzi, L. Gouda, S. Tirosh, Y. Bouhadana, A. Zaban, E. Mosconi, F. De Angelis, J. Phys. Chem. Lett. 2014, 5, 2662-2669.

[21] E. J. Juárez-Pérez, R. S. Sánchez, L. Badia, G. Garcia-Belmonte, Y. S. Kang, I. MoraSero, J. Bisquert, J. Phys. Chem. Lett. 2014, 5, 2390.

[22] J. Berry, T. Buonassisi, D. A. Egger, G. Hodes, L. Kronik, Y.-L. Loo, I. Lubomirsky, S. R. Marder, Y. Mastai, J. S. Miller, D. B. Mitzi, Y. Paz, A. M. Rappe, I. Riess, B. Rybtchinski, O. Stafsudd, V. Stevanovic, M. F. Toney, D. Zitoun, A. Kahn, D. Ginley, D. Cahen, Adv. Mater. 2015, 27, 5102.

[23] P. Schulz, E. Edri, S. Kirmayer, G. Hodes, D. Cahen, A. Kahn, Energy \& Environmental Science 2014, 7, 1377.

[24] E. M. Miller, Y. Zhao, C. C. Mercado, S. K. Saha, J. M. Luther, K. Zhu, V. Stevanovic, C. L. Perkins, J. v. de Lagemaat, Phys. Chem. Chem Phys. 2014, 16, 22122.

[25] A. Guerrero, E. J. Juarez-Perez, J. Bisquert, I. Mora-Sero, G. Garcia-Belmonte, Appl. Phys. Lett. 2014, 105, 133902.

[26] C.-S. Jiang, M. Yang, Y. Zhou, B. To, S. U. Nanayakkara, J. M. Luther, W. Zhou, J. J. Berry, J. v. de Lagemaat, N. P. Padture, K. Zhu, M. M. Al-Jassim, Nat. Commun. 2015, 6, 8397.

[27] O. Almora, I. Zarazua, E. Mas-Marza, I. Mora-Sero, J. Bisquert, G. Garcia-Belmonte, J. Phys. Chem. Lett. 2015, 6, 1645-1652.

[28] H.-S. Kim, I.-H. Jang, N. Ahn, M. Choi, A. Guerrero, J. Bisquert, N.-G. Park, J. Phys. Chem. Lett. 2015, 6, 4633-4639.

[29] W. Tress, N. Marinova, T. Moehl, S. M. Zakeeruddin, M. K. Nazeeruddin, M. Grätzel, Energy Environ. Sci 2015, 8, 225.

[30] V. Roiati, E. Mosconi, A. Listorti, S. Colella, G. Gigli, F. De Angelis, Nano Letters 2014, 14, 2168.

[31] J. M. Azpiroz, E. Mosconi, J. Bisquert, F. De Angelis, Energy Environ. Sci. 2015, 8, 2118.

[32] C. Eames, J. M. Frost, P. R. F. Barnes, B. C. O'Regan, A. Walsh, M. S. Islam, Nat. Commun. 2015, 6, 7497.

[33] M. Ledinský, P. Löper, B. Niesen, J. Holovský, S.-J. Moon, J.-H. Yum, S. De Wolf, A. Fejfar, C. Ballif, J. Phys. Chem. Lett. 2015, 6, 401. 
[34] U. B. Cappel, T. Daeneke, U. Bach, Nano Letters 2012, 12, 4925.

[35] W. H. Nguyen, C. D. Bailie, E. L. Unger, M. D. McGehee, Journal of the American Chemical Society 2014, 136, 10996.

[36] L. Badia, E. Mas-Marzá, R. S. Sánchez, E. M. Barea, J. Bisquert, I. Mora-Seró, APL Materials 2014, 2, 081507. 


\section{Ionic reactivity at contacts and aging of methylammonium lead triiodide perovskite solar cell}

Jordi Carrillo ${ }^{1}$, Antonio Guerrero ${ }^{1}$, Sara Rahimnejad ${ }^{1,2}$, Osbel Almora ${ }^{1}$, Isaac Zarazua ${ }^{1}$, Elena Mas-Marza ${ }^{1}$, Juan Bisquert $^{1,3}$, and Germà Garcia-Belmonte ${ }^{1, *}$

1 Institute of Advanced Materials (INAM), Universitat Jaume I, 12071 Castelló, Spain

2 Department of Chemistry, Faculty of Science Yadegar-e-Imam Khomeini (RAH) Shahre Rey Branch, Islamic Azad University, P.O. Box: 18155 144, Tehran, Iran 3 Department of Chemistry, Faculty of Science, King Abdulaziz University, Jeddah 21589, Saudi Arabia

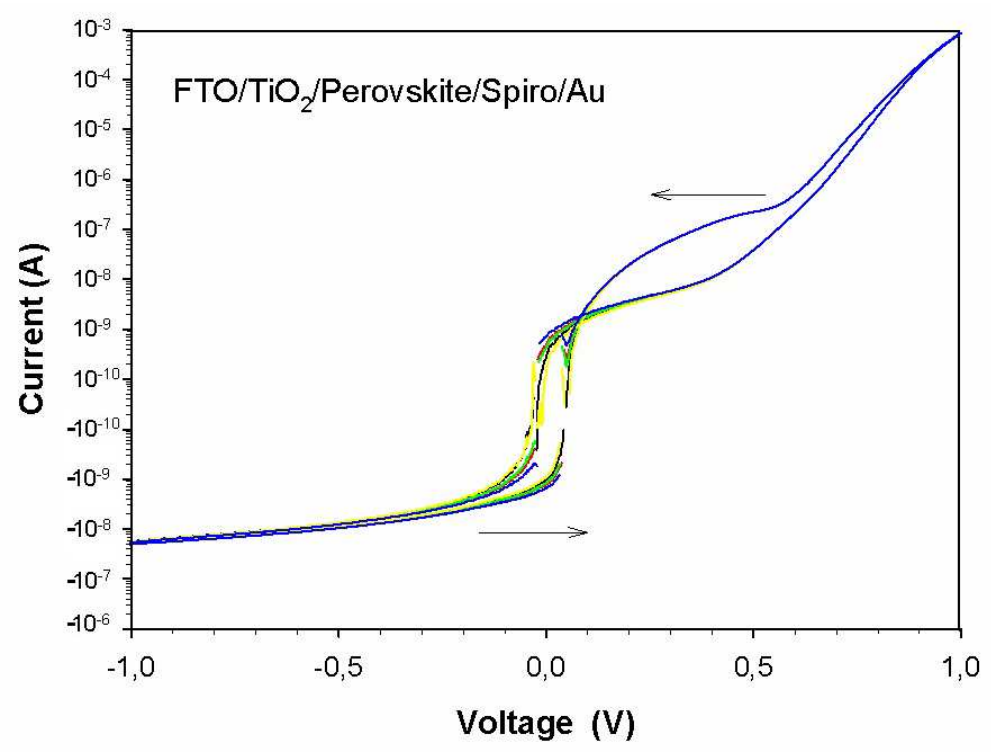

Figure S1. Successive five scans with negative poling (-1 V, $5 \mathrm{~min}$ ) showing no effect on the previous positive poling experiments. The negative peak appearing at positive poling is not observed here. 


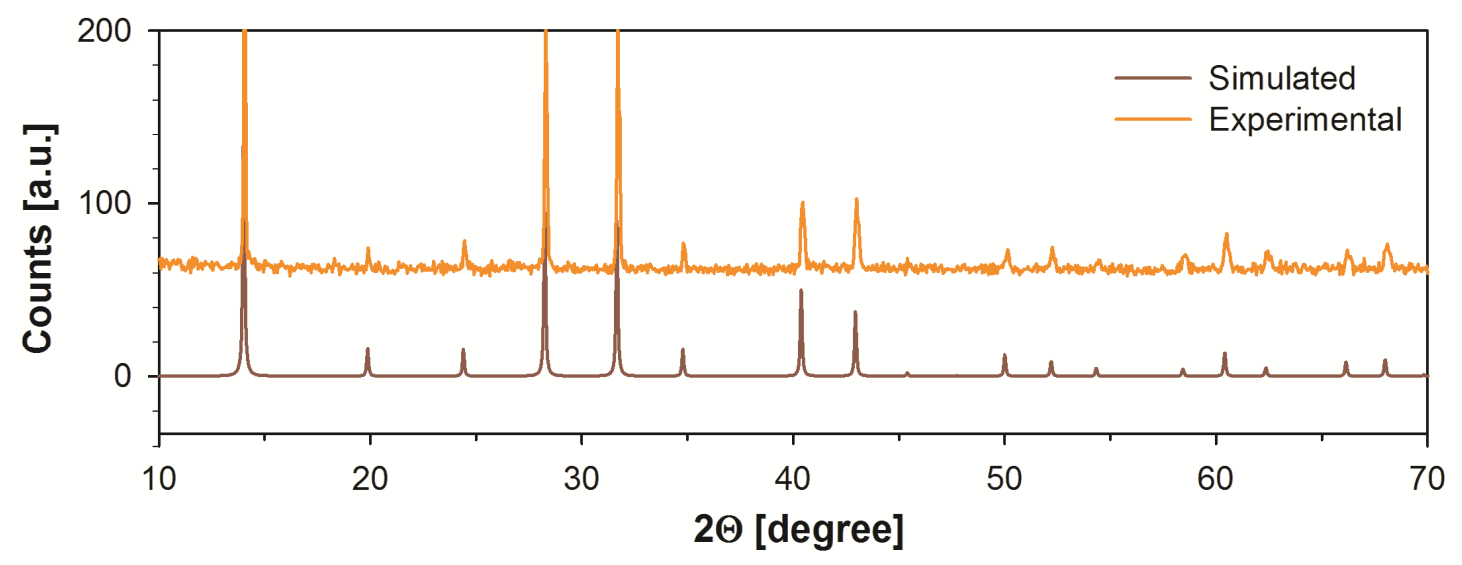

Figure $\mathrm{S} 2$. XRD pattern of $\mathrm{CH}_{3} \mathrm{NH}_{3} \mathrm{PbI}_{3}$ synthesised in this work. Simulated pattern has been calculated for the tetragonal phase obtained from a reported cif file [Journal of Physics: Condensed Matter 2014, 26, 284109]

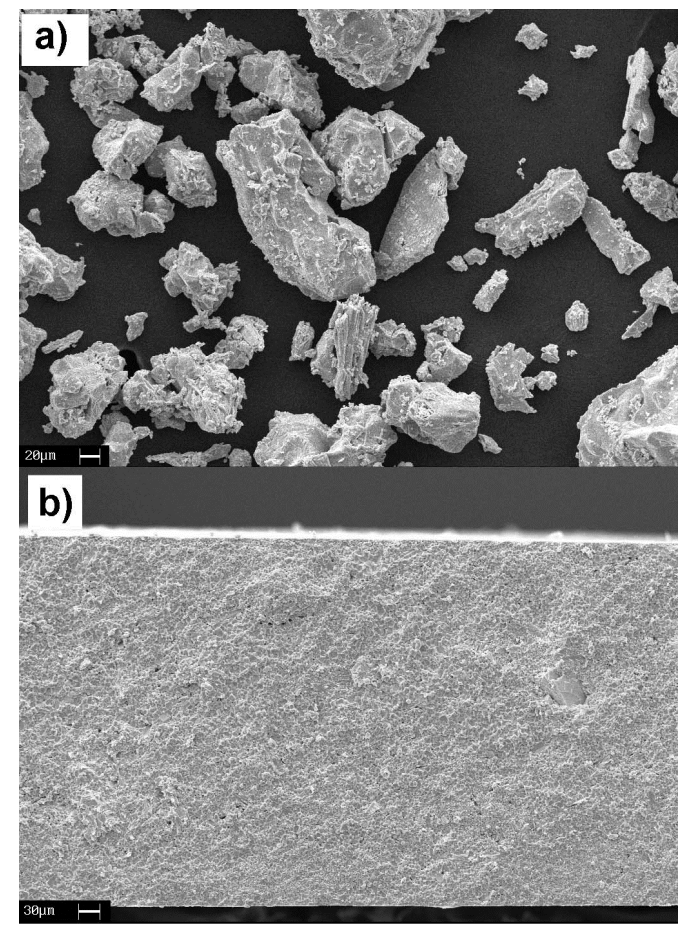


Figure S3. SEM images of a) $\mathrm{CH}_{3} \mathrm{NH}_{3} \mathrm{PbI}_{3}$ prepared in the synthesis of this manuscript and b) A cross-section of a perovskite pellet used for symmetric devices that shows thickness and morphology.

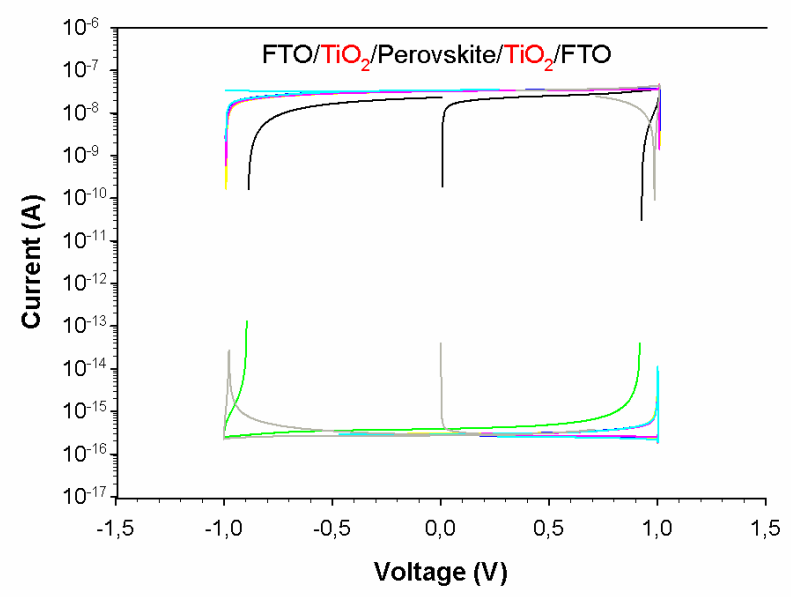

Figure S4. Current-voltage response of symmetric $\mathrm{FTO} / \mathrm{TiO}_{2} / \mathrm{MAPbI}_{3} / \mathrm{TiO}_{2} / \mathrm{FTO}$ samples showing the invariance of the response after positive $(1 \mathrm{~V})$ and negative $(-1 \mathrm{~V})$ polarization during $300 \mathrm{~s}$ and $900 \mathrm{~s}$. In all cases capacitive currents dominate the response.

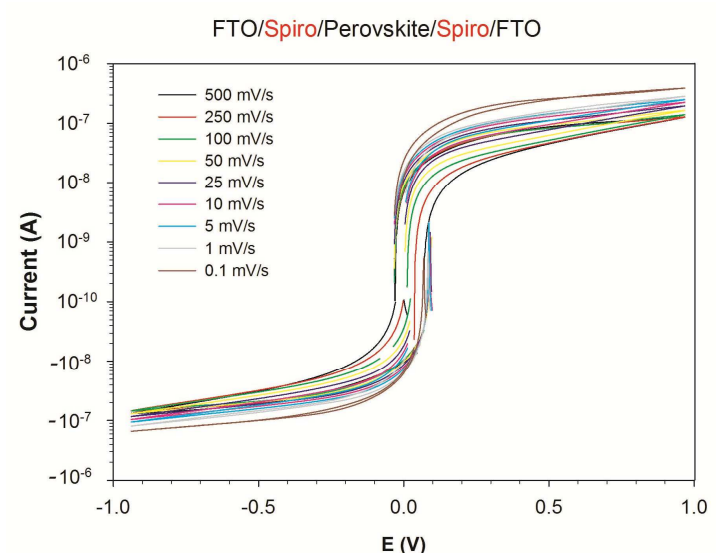

Figure S5. Current-voltage response of symmetric $\mathrm{Au} /$ spiro-OMeTAD/MAPbI $3 /$ spiro$\mathrm{OMeTad} / \mathrm{Au}$ samples with spiro-OMeTAD processed with lithium bis(trifluoromethylsulphonyl)imide. 


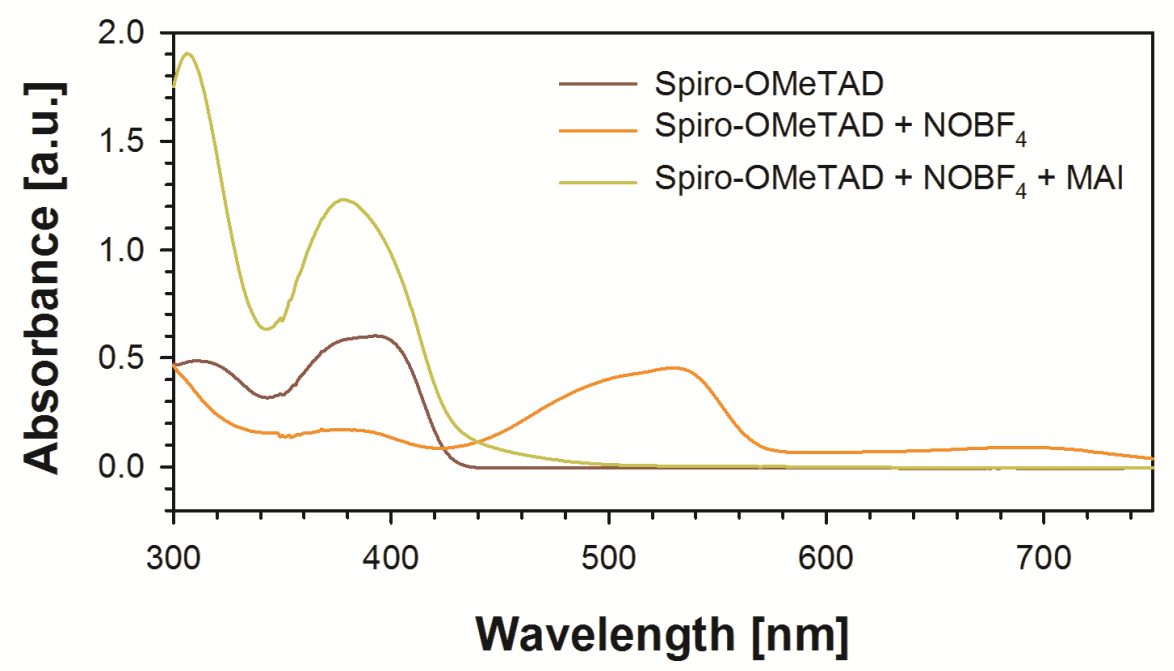

Figure S6. Absorption measurements of solutions containing spiro-OMeTAD (No LITFSI) at a concentration of $5 \mu \mathrm{M}$ in acetonitrile, oxidized solution using an equimolar concentration of $\mathrm{NOBF}_{4}$, and recovery of a neutral species by further reaction with MAI.

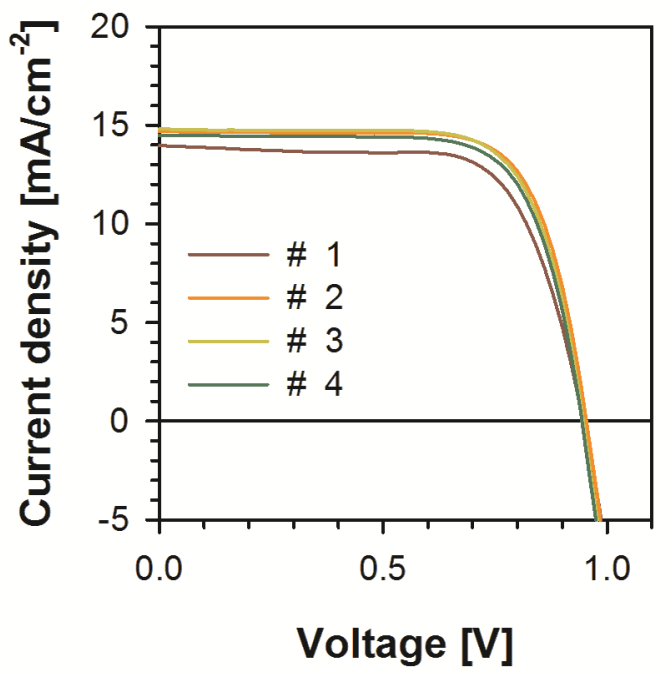

Figure S7. Current-voltage response of representative cells (as those used in Figure 1).

\begin{tabular}{|l|c|c|c|c|}
\hline & $\mathbf{J s c}\left(\mathbf{m A} / \mathbf{c m}^{-2}\right)$ & Voc (V) & FF (\%) & PCE (\%) \\
\hline Champion (measured) & 14.69 & 0.960 & 73.10 & 10.31 \\
\hline Average & 14.25 & 0.926 & 67.86 & 8.96 \\
\hline
\end{tabular}


